\title{
BMJ Open Exploring views and experiences of how infections are detected and managed in practice by nurses, care workers and manager's in nursing homes in England and Sweden: a survey protocol
}

N Carey (1) , ${ }^{1}$ Nouf Alkhamees, ${ }^{2}$ Anna Cox, ${ }^{1}$ Marta Sund-Levander, ${ }^{3}$ Pia Tingström, ${ }^{3}$ Freda Mold ${ }^{1}$

To cite: Carey N, Alkhamees N, $\operatorname{Cox} \mathrm{A}$, et al. Exploring views and experiences of how infections are detected and managed in practice by nurses, care workers and manager's in nursing homes in England and Sweden: a survey protocol. BMJ Open 2020;10:e038390. doi:10.1136/ bmjopen-2020-038390

- Prepublication history for this paper is available online. To view these files, please visit the journal online (http://dx.doi. org/10.1136/bmjopen-2020038390).

Received 09 March 2020

Revised 28 July 2020

Accepted 03 September 2020

Check for updates

(C) Author(s) (or their employer(s)) 2020. Re-use permitted under CC BY-NC. No commercial re-use. See rights and permissions. Published by BMJ.

${ }^{1}$ School of Health Sciences, University of Surrey, Guildford, UK

${ }^{2}$ College of Health and Rehabilitation Sciences, Princess Nourah Bint Abdulrahman University, Riyadh, Saudi Arabia

${ }^{3}$ Division of Nursing, Department of Medical and Health Sciences, Linkoping University, Linkoping, Sweden

Correspondence to

Dr N Carey;

n.carey@surrey.ac.uk

\section{ABSTRACT}

Introduction In order to avoid unnecessary hospital admission and associated complications, there is an urgent need to improve the early detection of infection in nursing home residents. Monitoring signs and symptoms with checklists or aids called decision support tools may help nursing home staff to detect infection in residents, particularly during the current COVID-19 pandemic. We plan to conduct a survey exploring views and experiences of how infections are detected and managed in practice by nurses, care workers and managers in nursing homes in England and Sweden.

Methods and analysis An international cross-sectional descriptive survey, using a pretested questionnaire, will be used to explore nurses, care workers and managers views and experiences of how infections are detected and managed in practice in nursing homes. Data will be analysed descriptively and univariate associations between personal and organisational factors explored. This will help identify important factors related to awareness, knowledge, attitudes, belief and skills likely to affect future implementation of a decision support tool for the early detection of infection in nursing home residents.

Ethics and dissemination This study was approved using the self-certification process at the University of Surrey and Linköping University ethics committee (Approval 2018/514-32) in 2018. Study findings will be disseminated through community/stakeholder/service user engagement events in each country, publication in academic peer-reviewed journals and conference presentations. A LAY summary will be provided to participants who indicate they would like to receive this information.

This is the first stage of a plan of work to revise and evaluate the Early Detection of Infection Scale (EDIS) tool and its effect on managing infections and reducing unplanned hospital admissions in nursing home residents. Implementation of the EDIS tool may have important implications for the healthcare economy; this will be explored in cost-benefit analyses as the work progresses.

\section{Strengths and limitations of this study}

- This is the first study to examine the views and views and experiences of how infections are detected and managed in practice by nurses, care workers and managers in nursing homes in England and Sweden.

- This is the first stage of a plan of work to revise and evaluate the Early Detection of Infection Scale (EDIS) tool and its effect on managing infections and reducing unplanned hospital admissions in nursing home residents.

- The survey will collect data across a range of nursing home settings, both urban and rural, in varying geographical areas in England and Sweden.

- Variations of data might occur between countries based on differences on support tool use across nursing home settings and different models of health and social care.

- The study will use a convenience approach to sampling, but the extent to which this will be representative of the nursing home workforce (ie, managers, care workers and nurses) in each country is not known.

\section{INTRODUCTION}

Global level predictions indicate that as a result of increased life expectancy around the world, $>2$ billion people will be aged $>65$ years by 2050 , with the number $>80$ years expected to reach 400 million by $2050 .^{12}$ The implications for managing the increased pressure this will have on healthcare resources and ability to meet patient demand, particularly for the population aged over 65 years, are profound.

Specific concerns have been raised about the care of nursing home residents $(\mathrm{NH})$ who often exhibit atypical signs and symptoms of infection, ${ }^{3}$ and are at increased risk of infection and unplanned hospital admissions resulting in clinical complications, increased 
mortality and extended length of stay. ${ }^{4-9}$ Unplanned hospital admissions account for more than one-third of all annual hospital admissions, and cost the National Health Service (NHS) in the UK over £11 billion each year. ${ }^{6}$ Unplanned hospital admissions cost the NHS $>£ 11$ billion, the US healthcare economy $>\$ 1.1$ trillion a year ${ }^{10}$ and the Swedish healthcare system $>$ SEK 36 trillion a year for people aged $>65$ years. ${ }^{11}$

The large increase in unplanned hospital admissions over the last decade which now account for $65 \%$ of all hospital bed days ${ }^{6} 812$ have exacerbated these concerns even more. This pressure is set to intensify further in line with the projected rise in people aged over 85 years and subsequent rise in the world population of nursing home residents. $^{21314}$

Nursing home residents around the world are at 1.4 times greater risk of emergency admission and have more than $50 \%$ unplanned hospital admissions compared with the general population aged 75 years or over. ${ }^{6} 891315$ However, studies show the hospitalisation of many nursing homes residents can be avoided ${ }^{7-9} 1416$ through rapid detection and more timely treatment. ${ }^{6915}$

There is therefore an urgent need to develop systems or tools to improve the early detection of infection (EDI), avoid unnecessary hospital admission and risk of complications in nursing home residents, ${ }^{691317}$ and help mitigate the spread of COVID- 19 during the current pandemic. ${ }^{18}$

Early indications suggests decision support tools (DST), which provide a systematic approach to monitoring cognitive and behavioural changes, can help ensure consistency, and more timely treatment. ${ }^{6} 15$ DST use for rapid EDI could prove helpful. ${ }^{319}$ Delegating DST use to nursing home staff, such as care workers, who are well positioned to recognise and communicate signs of deterioration, could reduce unplanned hospital admissions ${ }^{3} 19$ providing patient benefit.

Nursing home-oriented DSTs such as Stop and Watch ${ }^{19}$ rely primarily on observational assessment in order to define a resident who requires closer monitoring that will result in not only more frequent observations but also more frequent recording of vital signs. Another tool, the Early Detection of Infection Scale (EDIS), ${ }^{320-22}$ designed for completion by Swedish care workers, also includes assessment of body temperature. The assessment of body temperature uses a new approach based on the difference from baseline, the so-called 'DiffTemp' instead of predecided values for fever, ie, $>38^{\circ} \mathrm{C} .{ }^{23}$ Recording 'DiffTemp' as part of EDIS enables a more targeted approach focusing on specific aspects of behaviour and functional status. However, as EDIS is still undergoing ongoing development, it is yet to be to be widely adopted in Sweden. This is the first stage of a plan of work to evaluate a complex intervention, ${ }^{24}$ the intervention being the use of the EDIS tool in nursing homes in England and more widely in Sweden.

Given that ensuring quality and cost effectiveness is an increasingly essential requirement of modern healthcare delivery, exploration regarding the implementation of
DSTs that support early detection of infection in nursing homes is urgently required. It is recognised that the most challenging aspect of complex intervention research is implementation and 'normalisation' of the intervention. ${ }^{25}$ In this context, normalisation, as May and Finch ${ }^{25}$ explain, comprises not only understanding and evaluating the process by which interventions are embedded but also how they are sustained in practice. ${ }^{25}$

Therefore, this survey has an important role to play in gathering contextual information regarding how the EDIS intervention may be received and delivered in practice across diverse models of health and social care in England, and more widely in Sweden.

The aim of the study is to collect preliminary data exploring how infections are detected and managed in practice by nurses, care workers and managers in nursing homes in England and Sweden.

The study objectives are to:

1. Explore staff views on the types of infections that nursing home residents experience.

2. Explore staff views on how infection in nursing home residents is detected and managed.

3. Identify current challenges in detecting infection in nursing home residents and suggestions for improvement.

4. Explore the training and/or education of staff related to infection detection, and unmet training needs.

5. Examine knowledge, awareness and use of DSTs/ checklists available in practice, including infection detection.

This study builds on a systematic review of early interventions for people with frailty completed by the authors (NC/FM/PT/MS-L) that identified an urgent need to adopt innovate approaches to improving the care of frail older people. ${ }^{26}$

\section{METHODS/ DESIGN}

The 'Standard Protocol Items: Recommendations for Interventional Trials' statement, ${ }^{27}$ in parallel with the 'Survey reporting Guideline (SURGE)' guidance ${ }^{28}$ have been used to structure this protocol paper. Given the lack of formal register for survey research, adopting this approach supports transparency and reproducibility of the study protocol, ensuring quality and rigour in the research process. ${ }^{29}$

\section{SURVEY DESIGN}

A cross-sectional study descriptive survey will be used to collect data. In order to provide participants with flexibility in their method of response, the survey is available in two formats: (1) a paper-based version with accompanying pre-paid self-addressed envelope and (2) an online version accessed via a link to Qualtrics $\mathrm{XM},{ }^{30}$ a secure online survey platform. Given the complexity of reaching this population, ${ }^{31}$ and based on feedback received during survey development, it is anticipated that this approach 
will encourage nursing homes to participate. ${ }^{31}$ Allowing respondents to complete the survey at a convenient place and time should increase survey uptake, as well as supporting low-cost widespread distribution. ${ }^{29}$

\section{PARTICIPANTS}

A convenience sample of nurses, care workers and manager's employed in nursing homes in England $(n=2-3)$ and Sweden $(\mathrm{n}=2-3)$ (maximum 30 staff per home; approximately $90-100$ per country) will be invited to participate in the survey $(\mathrm{n}=200$ total). Currently, in the UK, there are around 550 nursing homes providing care to over $400 \mathrm{~K}$ people ${ }^{9}$ and 2600 nursing homes providing care to $100 \mathrm{~K}$ people in Sweden. ${ }^{32}$ Based on a $50 \%$ response rate the sample size $(n=90-100$ total $)$ is designed to ensure we collect enough data to support meaningful interpretation of the data ${ }^{29}$ and answer the research question.

In order to encourage study uptake, ${ }^{33}$ participants in England will be offered an incentive to be entered into an optional prize draw for $£ 100$ 'Love 2 Shop Vouchers' if they complete the survey.

\section{Patient and public involvement}

Preliminary discussions between nursing home managers, nurses and care workers in England and Sweden, and the study team in 2018 identified a high level of interest and enthusiasm towards DSTs that are practical, consistent, and acceptable to 'real world' nurses and care workers. They were overwhelmingly positive towards the previously developed EDIS tool for nursing home residents but were unsure of its potential benefits and or how it would work in practice.

In order to ensure the challenges and experiences of living and working in a residential nursing home were accurately reflected, the authors discussed the survey during a series of encounters with nursing home residents, care workers, a care home collaborative and regional interest groups in England and Sweden between June-December 2018.

PPI was invaluable in terms of developing the survey questions, participant information sheet and determining study recruitment and procedures. These discussions will be ongoing during data analysis, and interpretation. We will continue to work with our PPI members to help disseminate the findings through community/stakeholder/service user engagement events in each country and summarise the findings prior to public dissemination.

\section{DATA COLLECTION, MANAGEMENT AND ANALYSIS Procedure}

Managers in nursing homes in England and Sweden, where the team have established relationships, will initially be approached via telephone or email, to ascertain interest in the survey. Managers will be reassured that the location of their individual nursing home will not be reported or identifiable in the responses that participants provide. Those who express an interest in participating will be asked to confirm survey preference, and number of paper copies if required.

If no response is received, a follow-up telephone call or email will be made 2 weeks after initial contact. Where possible a member of the team will arrange to attend a staff meeting to talk about the survey and/or distribute surveys, either hardcopy or via a link to the online version. The team will maintain telephone/email contact with each home over the next 3 weeks to support data collection. Data collection will take place June 2019-June 2020. Online data will be collected by Qualtrics $\mathrm{XM}^{30}$, an online survey platform, selected as it allows flexibility in the device that is used to complete the survey, for example, mobile devices, desktop or laptop computers, alongside real-time data storage. Hard copy questionnaires will be returned in the stamp addressed envelope provided to the local University.

A pragmatic approach will be used to expand the range and number of nursing homes, and participants in each region if required until the target number of responses in each country $(n-50)$ is obtained. ${ }^{29}$

\section{THE QUESTIONNAIRE}

Development: an iterative approach to questionnaire development began with an initial 3 days development visit to Linköping in 2018 where team members (NC/FM/ $\mathrm{PT} / \mathrm{MS}-\mathrm{L}$ ) worked together to combine expertise in tool development, previous work in the area, ${ }^{319}$ and the literature. ${ }^{5934}$ This event resulted in the generation of a large pool of potential survey items, enabling the team to prioritise topics for inclusion and rephrase terms (such as pus/ oozing or rambling) to ensure each question adequately captured the topic. ${ }^{35}$

Service users were consulted during the early stage of the development as outlined above. ${ }^{36}$ To ensure questions accurately reflected the challenges and experience in nursing homes, and achieve face validity, ${ }^{29}$ a pretest version of the survey was reviewed by eight key stakeholders, including a local clinical commissioning group manager, care home representative groups and academic experts in the field. At this pretest stage stakeholders were asked to consider relevance of survey items; clarity of survey instructions and sequence of items. This process was repeated twice more until final agreement regarding the terms and expressions used was achieved.

The included questions were designed to specifically answer the research objectives. ${ }^{35}$ To minimise the risk of non-completion of survey items, we have aimed for fluidity of design and survey items, with largely tick box responses, and a relatively short completion time (approximately $10-15 \mathrm{~min}$ ). ${ }^{29} 35$

The draft survey was then translated in to Swedish (MS-L), back translated by an approved translation service provider (Språkservice Sverige $\mathrm{AB}$ ) and mapped against the original English version for linguistic accuracy and consistency in meaning. Following team discussion 
further final minor modifications were then made to Swedish version of the questionnaire.

\section{QUESTIONS}

The full questionnaire can be found in Additional file 1. The questionnaire comprises 29 questions over five sections.

\section{Section 1}

Section 1 comprises information about the survey, and a tick box to indicate consent.

\section{Section 2: working in a nursing home}

This section contains 12 questions, a mix of multiple response questions and closed questions regarding the types of infections that nursing home residents experience, frequency, actions that are taken, effect on residents' condition, level of confidence in their ability to manage infections, challenges in practice and suggested improvements.

\section{Section 3: education and training}

The third section consists of five questions exploring what if any training and or education has been undertaken, how useful this was, last training/education received; and what, if, any unmet training needs they have.

\section{Section 4: knowledge and awareness of DSTs}

This section has six questions that explore knowledge and awareness of DSTs/checklists, use of DSTs in practice and awareness of DSTs for infection detection and perceptions about their usefulness

\section{Section 5: general Information}

The final section contains six closed demographic questions regarding participants job title, working hours per week, years of employment in the current care home, years of experience, gender and age.

In this study, we will not ask respondents to state where they work thus assuring nursing homes remain anonymous. This is first to reassure respondents that they have an option to complete the questionnaire anonymously. ${ }^{29}$ Second as the focus of the study is about the use of DSTs across a range of geographical areas, the team agreed it was not important to link data back to specific nursing homes $^{29}$ in order to answer the research question.

\section{PRETESTING}

The questionnaire was piloted in both England and Sweden between January-March 2019. Respondents were asked to comment on ease and estimated time of completion, preference in terms of paper or online version and any difficulties they experienced. Twenty pilot surveys were distributed via one nursing home in each country, of which each home returned eight completed surveys (total $\mathrm{n}=16$ ).
Following piloting small changes were made to the ordering of questions to improve survey flow, sequencing of questions and duplicated items were removed from the multiple-choice questions. No issues arose in terms of process and/or completion, which was estimated to be 10-15 min.

Additionally, it was noted that the online version of the survey was more popular in Sweden. However, respondents from England preferred to use the paper version returned via a stamped address envelope to the University of Surrey.

\section{Data storage}

Data will be managed and processed in each country, and stored on password-protected computers, accessible only by team members and managed in line with current data protection regulations. Contact details disclosed by participants in order that they can receive a summary of study findings and/or if in England if they wish to be entered into the optional prize draw will be stored on a separate password-protected file in each country. Deidentified data from Sweden will be sent to the University of Surrey for analysis.

All files kept on the University of Surrey server, will only be accessible to the local research team. All data will be destroyed at the end of the archive period, in 10 years. Research data will be kept with strictest confidence in line with the EU General Data Protection Regulation (2018). ${ }^{37}$

\section{Data analysis}

Data, from hard copy questionnaires will be processed in each country. Electronic responses from Sweden and England will be stored in Qualtrics XM in the UK. Using a predetermined coding sheet data from Sweden and England will be combined in preparation for comparative analysis on SPSSv26. ${ }^{38}$ In order to minimise data inputting errors, a $10 \%$ check on data entry will be made in each country. ${ }^{29}$ Demographic data will be tabulated, and descriptive statistics undertaken on the sample in each country, and as whole. Further, statistical analysis (using parametric, non-parametric tests and a general linear modelling procedure) will be conducted where appropriate. Open-ended questions and free-text comments will be grouped into themes using content analysis to provide numerical counts of categories where appropriate ${ }^{39}$ and verified by a second member of the team.

\section{ETHICS AND DISSEMINATION Ethical considerations}

This study was approved using the self-certification process at the University of Surrey ${ }^{40}$ and by Linköping University ethics committee (Approval 2018/514-32) in 2018.

\section{Consent to participate}

Completion of the study will be entirely voluntary. Following provision of information regarding the 
survey, rationale and content, participant consent will be obtained (online supplemental additional files 1 and 2). The information and consent are positioned at the start of the survey, with participants requested to complete this before progressing to the survey questions. Contact details for the research team are provided, giving further opportunity to have any questions answered. Participants can withdraw at any time and advised if they wish to withdraw their data they must notify the research team within 2 weeks of completing a questionnaire. Participants in England will be offered an incentive to be entered into an optional prize draw for $£ 100$ 'Love 2 Shop Vouchers' if they complete the survey. All responses will be anonymous, unless participants disclose their contact details so they can receive a summary of the study, and/or if in England they wish to be entered into the optional prize draw.

\section{Dissemination of findings}

Study findings will be disseminated through community/ stakeholder/service user engagement events in each country, publication in academic peer-reviewed journals and conference presentations. Local and regional presentations will also be made to Kent Surrey Sussex Academic Health Science Network, Surrey Healthy Aging Research Partnership (SHARP) in the UK, and SKR, Sveriges Kommuner och Regioner (Sweden's Municipalities and Regions) in Sweden. A LAY summary will be provided to participants who indicate they would like to receive this information.

\section{DISCUSSION}

This paper presents a survey protocol that is designed to explore nurses, care workers and manager's views and experiences of how infections are detected and managed in practice by nurses, care workers and manager's in nursing homes in England and Sweden.

Evidence suggests that DSTs provide an opportunity to support a consistent approach to early detection of infection, enabling prompt action and treatment, thus avoiding emergency hospital admissions. ${ }^{914}$ Improving early detection of infection could minimise distress experienced by nursing home residents and their relatives when they are moved to an unfamiliar environment, avoiding associated complications, increased mortality and extended length of stay, ${ }^{8} 17$ and help mitigate the spread of COVID-19 during the current pandemic. ${ }^{18}$

This is the first stage of a plan of work to evaluate a complex intervention, ${ }^{24}$ the intervention being the use of the EDIS instrument in nursing homes in England and more widely in Sweden. Exploring nurses, care workers and managers' views and experiences of how infections are detected and managed in practice is an important first step to understanding enabling and reinforcing factors related to successful adoption, implementation and maintenance of the EDIS tool. This survey will identify the potential barriers, support and improve understanding of resources needed to implement and maintain EDIS in daily clinical practice not only in England, but also more widely in Sweden. ${ }^{24} 25$

Using principles of normalisation process theory, a conceptual framework to analyse implementation process, and inform recommendations to guide implementation work, ${ }^{25}$ a sequential programme of work is planned. It is well recognised that the most challenging aspect of complex intervention research is implementation and 'normalisation' of the intervention; therefore, this study has an important role to play in gathering contextual information regarding how the intervention may be received and delivered in practice in each country.

Working in collaboration with key stakeholders including service users, carers, managers and care home organisations, the results of the study will be used to explore the content of the EDIS tool, ${ }^{3}$ making any necessary revisions to ensure acceptability and suitability. We then plan to test the revised EDIS tool and its effect on managing infections and reducing unplanned admissions. ${ }^{24}$ It is anticipated that mixed methods including observation, focus groups and interviews will be used in future studies, assessing the impact of the EDIS tool, allowing triangulation of the data, and in-depth analysis. ${ }^{41}$ Given the important implications for the healthcare economy, cost-benefit analyses should also be considered as the work progresses.

Correction notice This article has been corrected since it was published. Affiliation updated for Dr Nouf Alkhamees.

Twitter N Carey @nmp_nicolacarey

Collaborators N/A.

Contributors NC, FM, MS-L and PT conceptualised the study. NC, FM and NA drafted the manuscript. AC, MS-L and PT reviewed and edited the manuscript. All authors read and approved the final manuscript.

Funding The project will be supported by awards from (1) School of Health Sciences and (2) Faculty of Health and Medical Sciences, University of Surrey.

Competing interests None declared.

Patient and public involvement Patients and/or the public were involved in the design, or conduct, or reporting, or dissemination plans of this research. Refer to the Methods section for further details.

Patient consent for publication Not required.

Provenance and peer review Not commissioned; externally peer reviewed.

Open access This is an open access article distributed in accordance with the Creative Commons Attribution Non Commercial (CC BY-NC 4.0) license, which permits others to distribute, remix, adapt, build upon this work non-commercially, and license their derivative works on different terms, provided the original work is properly cited, appropriate credit is given, any changes made indicated, and the use is non-commercial. See: http://creativecommons.org/licenses/by-nc/4.0/.

ORCID iD

N Carey http://orcid.org/0000-0003-2841-1760

\section{REFERENCES}

1 GBD 2016 Disease and Injury Incidence and Prevalence Collaborators. Global, regional, and national incidence, prevalence, and years lived with disability for 328 diseases and injuries for 195 countries, 1990-2016: a systematic analysis for the global burden of disease study 2016. Lancet 2017;390:1211-59. 
2 Holloway KA, Henry D. Who essential medicines policies and use in developing and transitional countries: an analysis of reported policy implementation and medicines use surveys. PLoS Med 2014;11:e1001724.

3 Tingström P, Milberg A, Rodhe N, et al. Nursing assistants: "he seems to be ill" - a reason for nurses to take action: validation of the Early Detection Scale of Infection (EDIS). BMC Geriatr 2015;15:122.

4 Shanley C, Whitmore E, Conforti D, et al. Decisions about transferring nursing home residents to hospital: highlighting the roles of advance care planning and support from local hospital and community health services. J Clin Nurs 2011;20:2897-906.

5 Grabowski DC, O'Malley AJ, Barhydt NR. The costs and potential savings associated with nursing home hospitalizations. Health Aff 2007;26:1753-61.

6 Smith P, Sherlaw-Johnson C, Ariti C, et al. Hospital admissions from care homes. The Health Foundation and NuffieldTrust, 2015.

7 Kirsebom M, Hedström M, Wadensten B, et al. The frequency of and reasons for acute Hospital transfers of older nursing home residents. Arch Gerontol Geriatr 2014;58:115-20.

8 Hallgren J, Aslan AKD, Dhal Asan AK. Risk factors for hospital readmission among Swedish older adults. Eur Geriatr Med 2018;9:603-11.

9 Wolters A, Santos AF, Llyod T. Emergency admissions to hospital from care homes: how often and what for? Health Foundation, 2019.

10 Centers of Medicare and Medicaid Services. Natioanl health expenditure accounts 2016. centers of Medicare and Medicaid services, 2018. Available: www.CMS.gov [Accessed 30th May 2018].

11 Havasi M. Costs of Healhcare for people aged over 65 in Sweden. Statistician Department of Finance and Governance SKR, Sveriges Kommuner och Regioner (Sweden's Municipalities and Regions)personal communication 2020.

12 Mkanta WN, Chumbler NR, Yang K, et al. Cost and Predictors of Hospitalizations for Ambulatory Care - Sensitive Conditions Among Medicaid Enrollees in Comprehensive Managed Care Plans. Health Serv Res Manag Epidemiol 2016;3:233339281667030-7.

13 Mcandrew RM, Grabowski DC, Dangi A, et al. Prevalence and patterns of potentially avoidable hospitalizations in the US long-term care setting. Int J Qual Health Care 2016;28:104-9.

14 Dahlberg L, Agahi N, Schön P, et al. Planned and unplanned hospital admissions and their relationship with social factors: findings from a national, prospective study of people aged 76 years or older. Health Serv Res 2018:53:4248-67.

15 The Medical Technology Group. Admissions of failure: the truth about unplanned NHS admissions in England, 2015. Available: www.mtg. org.uk;

16 Intrator O, Zinn J, Mor V. Nursing home characteristics and potentially preventable hospitalizations of long-stay residents. J Am Geriatr Soc 2004;52:1730-6.

17 NHS England. The framework for enhanced health in care homes, 2016.

18 OECD. Tackling coronavirus (COVID19): contributing to a gloabl effort, 2020. Available: http://www.oecd.org/coronavirus/en/ [Accessed 29th June 2020].

19 Ouslander JG, Bonner A, Herndon L, et al. The interventions to reduce acute care transfers (interact) quality improvement program an overview for medical directors and primary care clinicians in long term care. J Am Med Dir Assoc 2014;15:162-70.

20 Tingström $P$, Milberg A, Sund-Levander M. Early nonspecific signs and symptoms of infection in institutionalized elderly persons: perceptions of nursing assistants. Scand J Caring Sci 2010;24:24-31.

21 Sund-Levander M, Tingström P. Clinical decision-making process for early nonspecific signs of infection in institutionalised elderly persons: experience of nursing assistants. Scand J Caring Sci 2013;27:27-35.

22 Allemann $\mathrm{H}$, Sund-Levander $\mathrm{M}$. Nurses' actions in response to nursing assistants' observations of signs and symptoms of infections among nursing home residents. Nurs Open 2015;2:97-104

23 Levander MS, Grodzinsky E. Variation in normal ear temperature. Am J Med Sci 2017;354:370-8.

24 Craig P, Dieppe P, Macintyre S, et al. Developing and evaluating complex interventions: the new medical Research Council guidance. BMJ 2008;337:a1655.

25 May C, Finch T. Implementing, embedding, and integrating practices: an outline of normalization process theory. Sociology 2009;43:535-54.

26 Kidd T, Mold F, Jones C, et al. What are the most effective interventions to improve physical performance in pre-frail and frail adults? A systematic review of randomised control trials. BMC Geriatr 2019;19:184.

27 Chan A-W, Tetzlaff JM, Altman DG, et al. Spirit 2013: new guidance for content of clinical trial protocols. Lancet 2013;381:91-2.

28 Bennett C, Khangura S, Brehaut JC, et al. Reporting guidelines for survey research: an analysis of published guidance and reporting practices. PLoS Med 2010;8:e1001069.

29 Dillman D. Mail and Internet surveys: the tailored design method. Wiley, 1999.

30 Qualtrics software. Qualtrics and all other Qualtrics product or service names are registered trademarks or trademarks of Qualtrics, Provo, UT, USA, 2019. Available: https://www.qualtrics. com

31 Boynton PM, Wood GW, Greenhalgh T. Reaching beyond the white middle classes. BMJ 2004;328:1433-6.

32 Bergman MA, Johansson P, Lundberg S, et al. Privatization and quality: evidence from elderly care in Sweden. J Health Econ 2016;49:109-19.

33 Singer $\mathrm{E}$. The use and effects of incentives in surveys. Cham: Palgrave Macmillan, 2018.

34 Shanley C, Whitmore E, Conforti D, et al. Decisions about transferring nursing home residents to hospital: highlighting the roles of advance care planning and support from local hospital and community health services. J Clin Nurs 2011;20:2897-906.

35 Boynton PM, Greenhalgh T. Selecting, designing, and developing your questionnaire. BMJ 2004;328:1312-5.

36 NHS England. Patient and public participation policy, 2017. Available: https://www.england.nhs.uk/wp-content/uploads/2017/04/ ppp-policy.pdf [Accessed 24th Feb 2020].

37 Information Commissioners Office. Guide to the general data protection regulation (GDPR), 2018.

38 SPSS IBM Corp. Ibm SPSS statistics for windows, version 26.0 . Armonk, NY: IBM Corp, released 2018.

39 Field A. Discovering statistics using SPSS. 2nd ed. London: Sage, 2005.

40 University of Surrey. Self assessment form for ethics, 2018. Available: https://www.surrey.ac.uk/form/ethical-review-self-assessment

41 Cresswell JW. Research design: qualitative, quantitative, and mixed methods approaches. Sage, 2013. 